\title{
Search for supersymmetric partners of the tau lepton with CMS
}

\author{
Illia Babounikau*广 \\ $D E S Y$ \\ E-mail: ilya.bobovnikov@desy.de
}

Searches for supersymmetry are presented that target direct and indirect stau pair production. The analyses exploit the final states with two taus of opposite charge and significant missing transverse momentum. The results are based on a data set of proton-proton collisions, recorded by the CMS experiment at a center-of-mass energy of $13 \mathrm{TeV}$ and corresponding to an integrated luminosity of $36 \mathrm{fb}^{-1}$. Exclusion limits on parameters of simplified SUSY models are calculated. For direct $\widetilde{\tau}$ production we use three different $\widetilde{\tau}$ "chiral states": a purely left-handed $\tilde{\tau}$, a purely right-handed $\tilde{\tau}$ and maximal mixing between the right- and left-handed eigenstates. For indirect $\widetilde{\tau}$ production we consider simplified models of mass-degenerate chargino-neutralino and chargino pair production. Stau mass is an average value between the mass of the parent sparticles and LSP. For chargino pair production equal branching fractions are assumed for each of the two possible chargino decay chains and for chargino-neutralino production we set chargino mass to be equal to neutralino mass. Since the tau leptons can decay hadronically or leptonically, we target different decay channels in order to increase the number of signal events combining two analyses. The first analysis has both taus decay hadronically. The second has one tau decays to a lighter lepton and neutrinos, while the other one decays hadronically or first (second) tau decays to electron (muon) and neutrinos. No significant deviation from the SM in any signal region was observed. We exclude heavy neutralinos and charginos decaying through $\tilde{\tau}$ up to $725 \mathrm{GeV}$ for chargino-neutralino production and chargino decaying through $\tilde{\tau}$ up to $650 \mathrm{GeV}$ for chargino pair production. Direct $\tilde{\tau}$ production is not yet excluded due to low cross section. For left-handed stau of around 90 (125) GeV and the massless LSP we exclude 1.26 (1.34) times the expected SUSY cross-section.

The 39th International Conference on High Energy Physics (ICHEP2018)

4-11 July, 2018

Seoul, Korea

* Speaker.

${ }^{\dagger}$ on behalf of the CMS collaboration 


\section{Search strategy and results}

Searches for supersymmetry are presented that target direct and indirect stau pair production shown in Fig. 1 with the final states with two taus of opposite charge and significant missing transverse momentum [1].

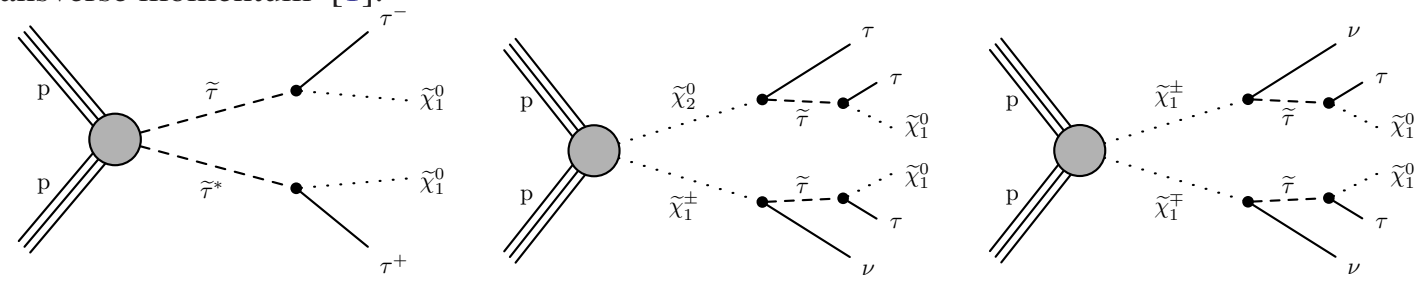

Figure 1: Diagrams for the simplified models studied in this paper: direct $\tilde{\tau}$ production followed by each $\tilde{\tau}$ decaying to a $\tau$ lepton and LSP (left), and chargino-neutralino (middle) and chargino pair (right) production.

We select events wtih an opposite charge pair of identified isolated leptons and without any additional leptons or jets originating from bottom quarks.

No significant deviation from the SM in any signal region was observed. All limits plots are shown in Fig. 2 and 3.
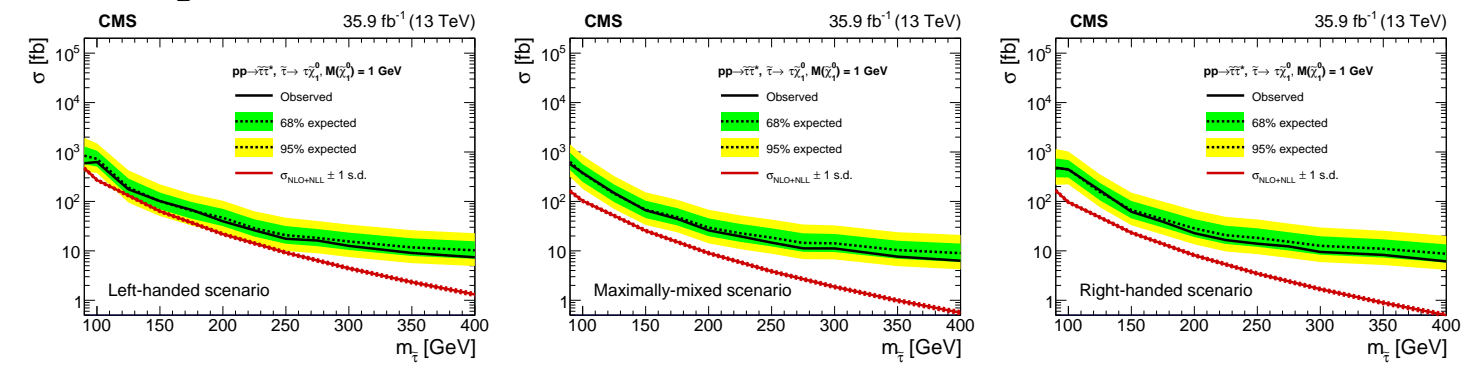

Figure 2: Excluded $\widetilde{\tau}$ pair production cross section as a function of the $\widetilde{\tau}$ mass for the left-handed (left), maximally-mixed (middle) and right-handed (right) scenarios, and with an LSP mass of $1 \mathrm{GeV}$.
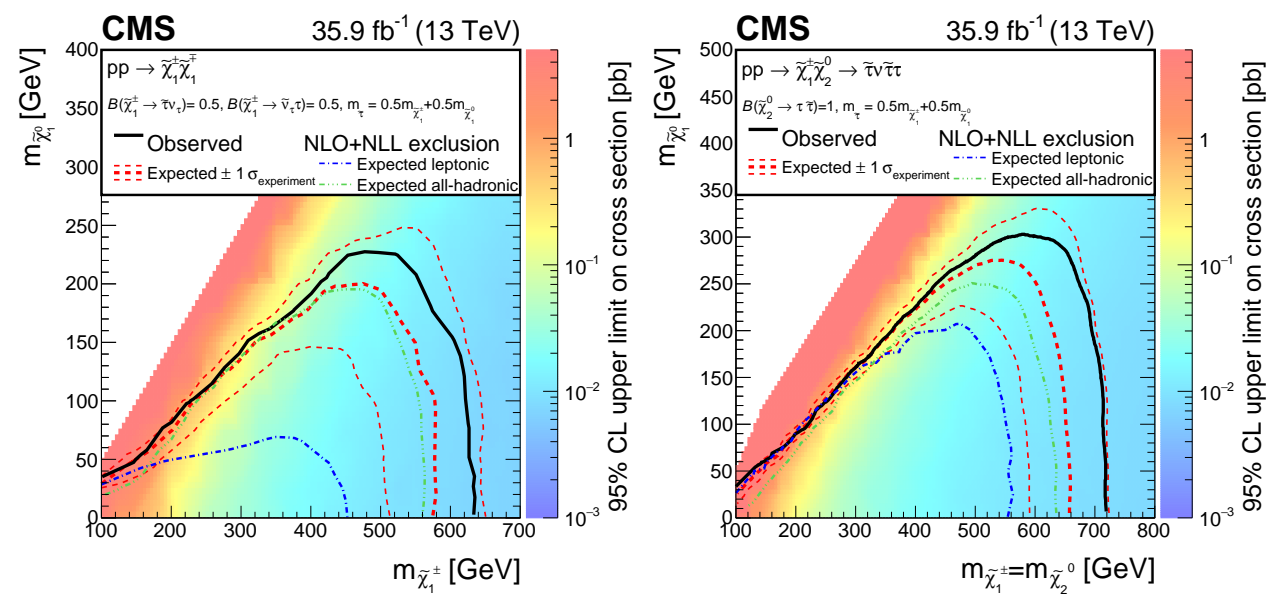

Figure 3: Exclusion limits at $95 \%$ for chargino pair (left) and chargino-neutralino (right) production with decays through $\tilde{\tau}$ to final states with leptons. The regions enclosed by the thick black curves represent the observed exclusion at $95 \%$, while the thick dashed red line indicates the expected exclusion at $95 \%$.

\section{References}

[1] A. M. Sirunyan et al. [CMS Collaboration], arXiv:1807.02048 [hep-ex]. 